\title{
Analytic modelling of tidal effects in the relativistic inspiral of binary neutron stars
}

\author{
Luca Baiotti, ${ }^{1,2}$ Thibault Damour, ${ }^{3,4}$ Bruno Giacomazzo,,${ }^{5,6,7}$ Alessandro Nagar, ${ }^{3}$ and Luciano Rezzolla ${ }^{7,8}$ \\ ${ }^{1}$ Institute of Laser Engineering, Osaka University, Suita, Japan \\ ${ }^{2}$ Yukawa Institute for Theoretical Physics, Kyoto University, Kyoto, Japan \\ ${ }^{3}$ Institut des Hautes Etudes Scientifiques, Bures-sur-Yvette, France \\ ${ }^{4}$ ICRANet, Pescara, Italy \\ ${ }^{5}$ Department of Astronomy, University of Maryland, College Park, Maryland USA \\ ${ }^{6}$ Gravitational Astrophysics Laboratory, NASA Goddard Space Flight Center, Greenbelt, Maryland USA \\ ${ }^{7}$ Max-Planck-Institut für Gravitationsphysik, Albert-Einstein-Institut, Potsdam, Germany \\ ${ }^{8}$ Department of Physics and Astronomy, Louisiana State University, Baton Rouge, Louisiana, USA
}

\begin{abstract}
To detect the gravitational-wave (GW) signal from binary neutron stars and extract information about the equation of state of matter at nuclear density, it is necessary to match the signal with a bank of accurate templates. We present the two longest (to date) general-relativistic simulations of equal-mass binary neutron stars with different compactnesses, $\mathcal{C}=0.12$ and $\mathcal{C}=0.14$, and compare them with a tidal extension of the effective-onebody (EOB) model. The typical numerical phasing errors over the $\simeq 22 \mathrm{GW}$ cycles are $\Delta \phi \simeq \pm 0.24 \mathrm{rad}$. By calibrating only one parameter (representing a higher-order amplification of tidal effects), the EOB model can reproduce, within the numerical error, the two numerical waveforms essentially up to the merger. By contrast, the third post-Newtonian Taylor-T4 approximant with leading-order tidal corrections dephases with respect to the numerical waveforms by several radians.
\end{abstract}

PACS numbers: 04.25.dk, 04.25.Nx, 04.30.Db, 04.40.Dg, 95.30.Sf, 97.60.Jd

Introduction. Inspiralling binary neutron stars (BNSs) are among the strongest sources of gravitational waves (GWs) and certain targets for the advanced and new-generation groundbased GW detectors LIGO/Virgo/GEO/ET [1]. These detectors will be sensitive to the inspiral GW signal up to GW frequencies of about $1 \mathrm{kHz}$, which are reached just before the merger. The late inspiral signal will be influenced by tidal interaction between the two neutron stars (NSs), which, in turn, encodes important information about the equation of state (EOS) of matter at nuclear densities. However, to reliably extract such information, both a large sample of numerical simulations and an analytical model of inspiralling BNSs which is able to reproduce them accurately, are needed. In this Letter we report on significant progress on this problem by presenting the longest (to date) simulations of merging equalmass BNSs and by showing how to use them to calibrate an effective-one-body (EOB) model of tidally interacting BNSs.

Numerical simulations of merging BNSs in full general relativity have reached a high-level of accuracy and have become more realistic (e.g., with the inclusion of magnetic fields) only recently [2-5]. The analytical description of tidallyinteracting binary systems has been initiated very recently [69], with the following two major results. First, the dimensionless quantity $k_{\ell}$ (Love number) in the (gravito-electric) tidal polarizability parameter $G \mu_{\ell} \equiv 2 k_{\ell} R^{2 \ell+1} /(2 \ell-1)$ !! measuring the relativistic coupling (of multipolar order $\ell$ ) between a NS of radius $R$ and the external gravitational field in which it is embedded has been found to be a strongly decreasing function of the compactness parameter $\mathcal{C} \equiv G M /\left(c^{2} R\right)$ of the NS. Second, a comparison between the numerical computation of the binding energy of quasi-equilibrium circular sequences of BNSs [10] and the EOB description of tidal effects [9] has suggested that higher-order post-Newtonian (PN) corrections to tidal effects effectively increase by a factor of order two the tidal polarizability of close NSs. The main aim of this paper is to extend the domain of applicability of the EOB method [11], from the inspiralling binary black hole $(\mathrm{BBH})$ case (for which it recently provided a very accurate analytic description [12,13]), to the yet unexplored case of inspiralling BNSs. To this aim we have performed two accurate and longterm BNS simulations covering approximately $20-22 \mathrm{GW}$ cycles of late inspiral, and we will show that they can be reproduced accurately almost up to the merger by a new tidal extension of the EOB model.

Tidal corrections in the EOB approach. We recall that the EOB formalism [11] replaces the PN-expanded two-body dynamics by a resummed description with, in particular, a Hamiltonian of the form: $H_{\mathrm{EOB}} \equiv M c^{2} \sqrt{1+2 \nu\left(\hat{H}_{\mathrm{eff}}-1\right)}$, where $M \equiv M_{A}+M_{B}$ is the total mass and where $\nu \equiv M_{A} M_{B} /\left(M_{A}+M_{B}\right)^{2}$ is the symmetric mass ratio. Here the "effective Hamiltonian" $\hat{H}_{\text {eff }}$ is a simple function of the momenta and it incorporates the relativistic gravitational attraction mainly through the so-called "EOB radial potential" $A(r)$. The structure of $A(r)$ is remarkably simple at 3PN: $A^{3 \mathrm{PN}}(r)=1-2 u+2 \nu u^{3}+a_{4} \nu u^{4}$, where $a_{4}=94 / 3-(41 / 32) \pi^{2}$, and $u \equiv G M /\left(c^{2} r_{A B}\right)$. An excellent description of BBHs has been found to be given by [12] $A^{0}(r)=P_{5}^{1}\left[1-2 u+2 \nu u^{3}+a_{4} \nu u^{4}+a_{5} \nu u^{5}+a_{6} \nu u^{6}\right]$, where $P_{m}^{n}$ denotes an $(n, m)$ Padé approximant and where values of the coefficient $a_{5}=-6.37, a_{6}=+50$ provide a very good agreement between EOB and numerical-relativity (NR) waveforms for BBHs [12] (the results presented here are insensitive to this choice as long as $a_{5}$ and $a_{6}$ are chosen in a well defined range). Ref. [9] suggested to include tidal effects as corrections both to the radial potential and to the waveform (and radiation reaction). The tidally corrected radial potential reads $A(u)=A^{0}(u)+A^{\text {tidal }}(u)$, where

$$
A^{\text {tidal }}=\sum_{\ell \geq 2}-\kappa_{\ell}^{T} u^{2 \ell+2} \hat{A}_{\ell}^{\text {tidal }}(u) .
$$


Here $\kappa_{\ell}^{T} u^{2 \ell+2}$ describes the leading-order (LO) tidal interactions. It is a function of the two masses, of the two compactnesses $\mathcal{C}_{A, B}$, and of the two (relativistic) Love numbers $k_{\ell}^{A, B}$

$$
\kappa_{\ell}^{T}=2 \frac{M_{B} M_{A}^{2 \ell}}{\left(M_{A}+M_{B}\right)^{2 \ell+1}} \frac{k_{\ell}^{A}}{\mathcal{C}_{A}^{2 \ell+1}}+\left\{A \leftrightarrow{ }_{B}\right\} .
$$

The additional factor $\hat{A}_{\ell}^{\text {tidal }}(u)$ in Eq. (1) represents the effect of higher-order relativistic contributions to the tidal interactions: next-to-leading order (NLO), next-to-next-to-leading order (NNLO), etc. A number of different prescriptions can be considered for the correcting tidal factor $\hat{A}_{\ell}^{\text {tidal }}$ and these will be presented in a longer companion work [14]. Here, we will limit ourselves to an $\ell$-independent, "Taylor-expanded" expression $\hat{A}_{\ell}^{\text {tidal }}(u) \equiv 1+\bar{\alpha}_{1} u+\bar{\alpha}_{2} u^{2}$ [9], where $\bar{\alpha}_{n}$ are pure numbers in the equal-mass case, but functions of $M_{A}$, $\mathcal{C}_{A}$, and $k_{\ell}^{A}$ in the general case. The analytical value of the $(\ell=2) 1$ PN coefficient $\bar{\alpha}_{1}$ has been reported in [9] (and recently confirmed in [15]). In the equal-mass case, it yields $\bar{\alpha}_{1}=1.25$. We will use this analytical value in the following and use our simulations to constrain the value of the $2 \mathrm{PN}$ coefficient $\bar{\alpha}_{2}$. Similarly, one takes into account an $\ell=2$ tidal correction to the waveform and radiation reaction, as given at LO in Sec. V of [9]. Additional coefficients parametrizing higher-order tidal relativistic contributions in the waveform and radiation reaction (such as $\beta_{1}$ in Eq. (71) of [9]), were found to have a small effect [14] and will be neglected here. In principle, tidal effects can also be accounted for via modifications of one of the non-resummed PN models, such as the Taylor-T4 one; see below for its comparison with the NR results.

In order to measure the influence of tidal effects, it is useful to consider the "phase acceleration" $\dot{\omega} \equiv d \omega / d t \equiv d^{2} \phi / d t^{2}$, where $\phi \equiv \phi_{22}$ is the phase of either the curvature or of the metric $\ell=m=2 \mathrm{GWs}$. The function $\dot{\omega}(\omega)$ is independent of the two "shift ambiguities" that affect the GW phase $\phi(t)$, namely the shifts in time and phase, and thus a useful intrinsic measure of the quality of the waveform [17]. However, here we use another diagnostic to measure the phase acceleration, namely the dimensionless function

$$
Q_{\omega}(\omega)=\frac{d \phi}{d \ln \omega}=\frac{\omega d \phi / d t}{d \omega / d t}=\frac{\omega^{2}}{\dot{\omega}} .
$$

Numerical Simulations. They were performed with the Cactus-Carpet-Whisky [18] codes and, in essence, we use the same gauges and numerical methods already discussed in [2]. As initial data we use quasi-equilibrium irrotational binaries generated with the multi-domain spectral-method code LORENE, within a conformally-flat spacetime metric [19]. The EOS of the initial data is the polytropic one $p=K \rho^{\Gamma}$, where $p, \rho, K=123.6$, and $\Gamma=2$ are the pressure, restmass density, polytropic constant, and adiabatic index, respectively (in units where $c=G=M_{\odot}=1$ ). The evolutions are instead performed with either a polytropic EOS or an "ideal-fluid" one, $p=\rho \epsilon(\Gamma-1)$, where $\epsilon$ is the specific internal energy; the differences in phasing introduced by the different EOSs are of order $\pm 0.13 \mathrm{rad}$ [14]. Because

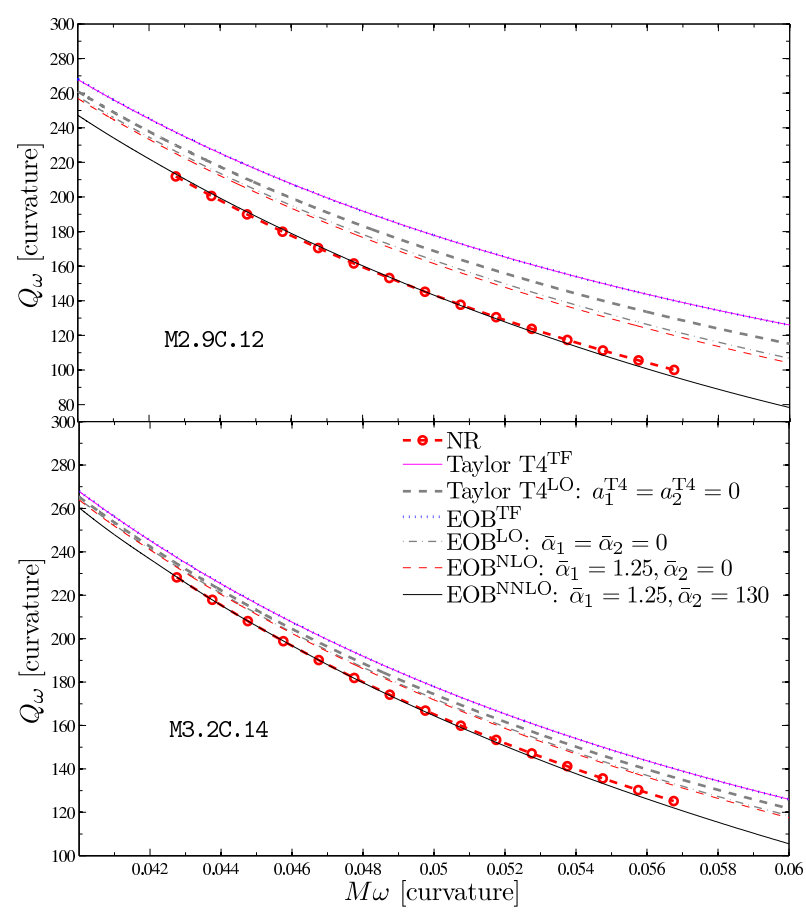

FIG. 1. Comparison of the EOB $Q_{\omega}$ curves for different choices of the effective tidal amplification factor $\hat{A}_{\ell}^{\text {tidal }}(u)=1+\bar{\alpha}_{1} u+\bar{\alpha}_{2} u^{2}$, with the corresponding NR ones (dashed lines with open circles) for the two binaries considered. The dotted line corresponds to the "tidal-free" ( or "point-mass") EOB, namely when ignoring tidal effects. The figure also includes two Taylor-T4 models: tidal-free, and augmented by LO tidal effects.

the stellar compactness represents the most important parameter determining the size of tidal effects, we have considered two different (equal-mass) binaries having total ArnowittDeser-Misner (baryonic) mass of either $2.69(2.89) M_{\odot}$ or $3.00(3.25) M_{\odot}$, thus with compactnesses $\mathcal{C} \equiv \mathcal{C}_{A}=\mathcal{C}_{B}=$ 0.12 or $\mathcal{C}=0.14$. The dominant $(\ell=2)$ tidal parameters for the two compactnesses $\mathcal{C}=0.12(0.14)$ are found to be [7], respectively, $k_{2} \equiv k_{2}^{A}=k_{2}^{B}=0.00969$ (0.07894), and therefore $\kappa_{2}^{T}=496.01$ (183.81). Hereafter the two binaries will be referred to as M2.9C.12 and M3.2C.14, respectively. The number of refinement levels and their resolutions are the same as those in [2], but the initial coordinate separation between the stellar centers is $60 \mathrm{~km}$, i.e. considerably larger than the one considered in [2]. This yields about 10 orbits before merger, thus the two longest BNS waveforms produced to date.

Discussion. We start our comparison between the NR results and the EOB ones by showing in Fig. 1 the $Q_{\omega}$ diagnostics for various possible LO/NLO tidal models and for scaled GW frequencies $M \omega \lesssim 0.06$ [i.e. up to 3 (5) GW cycles before merger for the M2.9C.12 (M3.2C.14) binary]. [We have estimated the NR $Q_{\omega}$ by fitting each NR phase evolution on a (scaled) frequency interval $I=[0.043,0.057]$ with an analytical expression that reproduces at lower order the behavior expected from the PN approximation, thereby filtering out the amplified numerical noise coming from the two time 

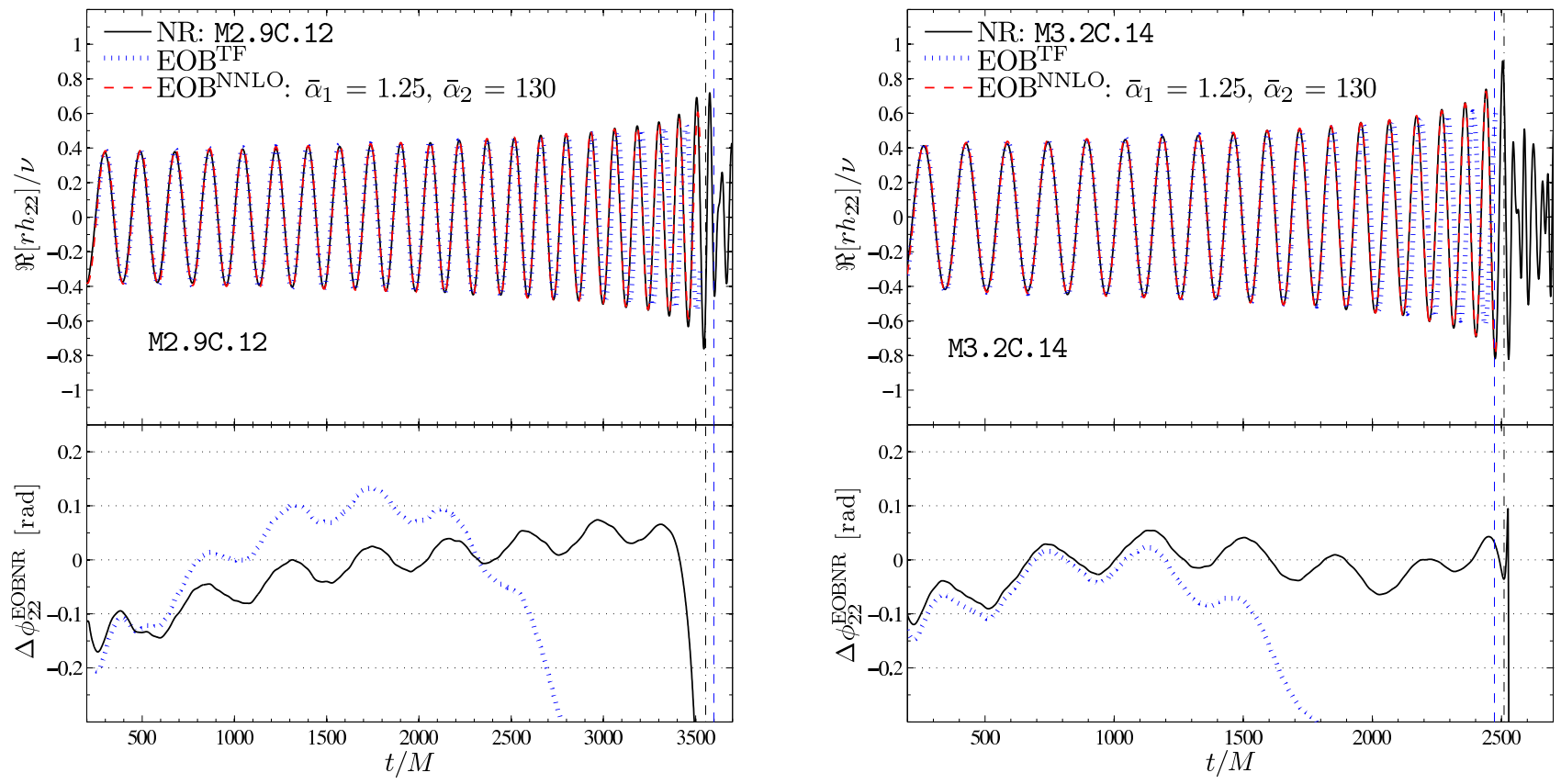

FIG. 2. Comparison between NR and EOB phasing for the M2.9C.12 (left panels) and M3.2C.14 (right panels) binaries. The top panels show the real parts of the $h_{22}$ waveforms, while the bottom panels show the corresponding phase differences. Note the excellent agreement almost up to the time of merger (vertical dashed and dot-dashed lines) and the very large errors when tidal effects are neglected (dotted line).

derivatives in $\dot{\omega} \equiv d^{2} \phi / d t^{2}$ (more details will be presented in [14])]. The first thing to note is that both the tidal-free $\mathrm{EOB}$ model (EOB ${ }^{\mathrm{TF}}$, dotted line) and the EOB model including only $\mathrm{LO}$ tidal corrections $\left(\mathrm{EOB}^{\mathrm{LO}}\right.$, dot-dashed line) are clearly unable, both for the M2 .9C.12 (upper panel) and the M3 . 2C. 14 binaries (lower panel), to match the corresponding NR curves (dashed line with open circles). The dephasing accumulated over the frequency interval $I \Delta_{I} \phi^{\mathrm{EOBNR}} \equiv$ $\int_{I}\left(Q_{\omega}^{\mathrm{EOB}}-Q_{\omega}^{\mathrm{NR}}\right) d \ln \omega$, by the $\mathrm{EOB}^{\mathrm{LO}}$ model relative to the $\mathcal{C}=0.12(0.14) \mathrm{NR}$ data is about $5.5(2.0) \mathrm{rad}$. This is much larger than the NR phasing error, related to a finiteradius extraction and EOS dependence, estimated to be $\Delta \phi=$ $\pm 0.24[14]$.

The inclusion of the NLO 1PN tidal effect $\left(\bar{\alpha}_{1}=1.25\right.$ [9]) only slightly reduces these dephasings to about $4.9(1.8) \mathrm{rad}$ $\left(\mathrm{EOB}^{\mathrm{NLO}}\right.$ curves in Fig. 11). This clearly indicates the need for NNLO (2PN and higher) tidal effects. We then found that choosing $\bar{\alpha}_{2} \approx 130$, yields a good match between the $Q_{\omega}$ curves (solid line, $\mathrm{EOB}^{\mathrm{NNLO}}$ ) and the NR data (dashed line with open circles) for both binaries, with a corresponding phase difference $\Delta_{I} \phi^{\mathrm{EOBNR}} \approx 0.1 \mathrm{rad}$. The value $\bar{\alpha}_{2} \approx 130$ is probably only an effective description of higher-order relativistic tidal effects. Moreover, the precise value of $\bar{\alpha}_{2}$, or more generally of the amplification factor $\hat{A}_{\ell}^{\text {tidal }}(u)$, is sensitive to the numerical truncation error. When considering resolution-extrapolated GWs [14], we found a smaller value of $\bar{\alpha}_{2}$, which is compatible with the estimate obtained using the binding energy of circular BNSs [10].

Figure 1 also reports the $Q_{\omega}$ diagnostics obtained when using two versions of the Taylor-T4 approximant: the tidalfree model ( $\mathrm{T} 4{ }^{\mathrm{TF}}$, magenta, upper solid line), and the Taylor-
$\mathrm{T} 4^{\mathrm{LO}}$ one (thick-dashed line). The latter model includes only the LO tidal effects [6], i.e. the LO tidal contribution $a^{\text {tidal }}(x) \propto \kappa_{2}^{T} x^{5}$ to $d x / d t$ [where $x \equiv(M \omega / 2)^{2 / 3}$; see [8] and Eqs. (86)-(88) of [9]]. Note that the tidal-free Taylor-T4 $Q_{\omega}$ curve nearly coincides with the tidal-free EOB one, with a dephasing $\Delta_{I} \phi^{\mathrm{T} 4 \mathrm{EOB}}=0.013 \mathrm{rad}$. On the other hand, the $I$ integrated dephasings between the $\mathrm{T} 4^{\mathrm{LO}}$ description and the $\mathrm{NR}$ results are rather large, namely $\Delta_{I} \phi^{\mathrm{T} 4 \mathrm{NR}}=6.96(2.53)$ $\operatorname{rad}$ for $\mathcal{C}=0.12(0.14)$. We have investigated whether a suitable PN-amplification factor $\hat{a}^{\mathrm{tidal}}(x)=1+a_{1}^{\mathrm{T} 4} x+a_{2}^{\mathrm{T} 4} x^{2}$ of $a^{\text {tidal }}(x)$ might accurately reproduce the NR data, but we found that this was not possible with a single choice of $\hat{a}^{\text {tidal }}(x)$ for the two simulations [14]. The latter result suggests that the EOB modelling of tidal effects may be more robust than the corresponding Taylor-T4 one

We next consider the comparison of the waveforms in the time domain and over the full inspiral up to the merger. This is shown in Fig. 2. whose left panels refer to the M2 . 9 C . 12 binary and the right ones to M3.2C.14. The top parts compare the (real part) of the $\mathrm{EOB}^{\mathrm{NNLO}}$ (with $\bar{\alpha}_{1}=1.25, \bar{\alpha}_{2}=$ $130)$ and NR metric $h_{22}$ waveforms, while the bottom panels show the corresponding phase differences, $\Delta \phi^{\mathrm{EOBNR}}(t) \equiv$ $\phi^{\mathrm{EOB}}(t)-\phi^{\mathrm{NR}}(t)$ (suitably shifted in time and phase à la [16]). The two vertical lines indicate two possible markers of the "time of the merger"; more specifically, the dashed lines refer to the NR merger, defined as the time at which the modulus of the metric NR waveform reaches its first maximum, while the vertical dash-dotted line represents the EOB estimate of the "formal" contact [9]. Figure 2]clearly shows that the agreement in the time domain between the analytic EOB description and the numerical one is extremely good essen- 
tially up to the merger, with a phase error which is well within the estimated error bar. More precisely: (i) in the M3 . 2C.14 case, the phase difference $\Delta \phi^{\mathrm{EOBNR}}(t)$ varies between -0.1 rad in the early inspiral and $+0.04 \mathrm{rad}$ at the NR merger; (ii) in the M3 . $2 \mathrm{C} .12$ case, $\Delta \phi^{\mathrm{EOBNR}}(t)$ varies between $-0.15 \mathrm{rad}$ in the early inspiral and $-0.15 \mathrm{rad}$ as late as $130 M$ (i.e. approximately $1.5 \mathrm{GW}$ cycles) before the NR merger. For the latter binary, it is only just before the NR merger that the dephasing becomes of order $1 \mathrm{rad}$.

Conclusions. We have presented the first NR-EOB comparison of the GWs emitted during the inspiral of relativistic BNSs. In particular, we have analyzed the longest to date numerical simulations of equal-mass, irrotational BNSs with two different compactnesses. We found that tidal effects are significantly amplified by higher-order relativistic corrections (2PN and higher) and that the inclusion of such corrections is necessary for an accurate phasing of the GW signal. Such an amplification is equivalent to a (distance-dependent) effective increase of the Love numbers. When a single choice for the unique free parameter in the NNLO term is made, the EOB model is able to reproduce the two NR phase evolutions well within the estimated NR error and essentially up to merger (and in fact up to merger in the $\mathcal{C}=0.14$ case). By contrast, we have shown that the use of the Taylor-T4 PN approximant considered in [8] leads to phase disagreements (over the frequency interval $I=[0.043,0.057]) \Delta_{I} \phi^{\mathrm{T} 4 \mathrm{NR}}=6.96(2.53)$ $\operatorname{rad}$ for $\mathcal{C}=0.12(0.14)$.

The work reported here provides the first evidence that an accurate analytic modelling of the late inspiral of tidally interacting BNSs is possible, thereby opening the possibility to extract reliable information on the EOS of matter at nuclear densities from the data of the forthcoming advanced GW detectors. These encouraging results, however, also call for a continued synergy between more accurate numerical simulations (notably exploring different mass ratios) and higherorder analytic results.

Acknowledgments. We thank the developers of Cactus and Carpet for their continuous improvements. The simulations were performed on Ranger (TACC/TG-MCA02N014) and Damiana (AEI). This work was supported by the DFG grant SFB/Transregio 7, by "CompStar", a Research Networking Programme of the ESF, by the JSPS Grant-in-Aid for Scientific Research (19-07803), by the MEXT Grant-in-Aid for Young Scientists (22740163), and by NASA (NNX09AI75G).
[1] N. Andersson, et al., Gen. Rel. Grav. in press arXiv:0912.0384.

[2] L. Baiotti, B. Giacomazzo, and L. Rezzolla, Phys. Rev. D 78, 084033 (2008).

[3] T. Yamamoto, M. Shibata, and K. Taniguchi, Phys. Rev. D 78, 064054 (2008); L. Baiotti, B. Giacomazzo, and L. Rezzolla, Class. Quantum Grav. 26, 114005 (2009); L. Rezzolla, L. Baiotti, B. Giacomazzo, D. Link, and J.-A. Font, Class. Quantum Grav. 27, 114105 (2010).

[4] M. Anderson, et al., Phys. Rev. Lett. 100, 191101 (2008); Y. T. Liu, S. L. Shapiro, Z. B. Etienne, and K. Taniguchi, Phys. Rev. D78, 024012 (2008); B. Giacomazzo, L. Rezzolla, and L. Baiotti, MNRAS 399, L164 (2009); B. Giacomazzo, L. Rezzolla, and L. Baiotti, arXiv:1009.2468.

[5] J. S. Read, C. Markakis, M. Shibata, K. Uryu, J. D. E. Creighton, and J. L. Friedman, Phys. Rev. D 79, 124033 (2009); K. Kiuchi, Y. Sekiguchi, M. Shibata, and K. Taniguchi, Phys. Rev. D 80, 064037 (2009); K. Kiuchi, Y. Sekiguchi, M. Shibata, and K. Taniguchi, Phys. Rev. Lett. 104, 141101 (2010).

[6] E. E. Flanagan, T. Hinderer, Phys. Rev. D77, 021502 (2008).

[7] T. Hinderer, Astrophys. J. 677, 1216 (2008); T. Damour and A. Nagar, Phys. Rev. D 80, 084035 (2009); T. Binnington and E. Poisson, Phys. Rev. D80, 084018 (2009).

[8] T. Hinderer, B. D. Lackey, R. N. Lang et al., Phys. Rev. D81,
123016 (2010).

[9] T. Damour and A. Nagar, Phys. Rev. D 81, 084016 (2010).

[10] K. Uryu, F. Limousin, J. L. Friedman, E. Gourgoulhon, and M. Shibata, Phys. Rev. D80, 124004 (2009).

[11] A. Buonanno and T. Damour, Phys. Rev. D 59, 084006 (1999); Phys. Rev. D 62, 064015 (2000). T. Damour, Phys. Rev. D 64, 124013 (2001).

[12] T. Damour and A. Nagar Phys. Rev. D 79, 081503 (R) (2009).

[13] A. Buonanno et al., Phys. Rev. D79, 124028 (2009).

[14] L. Baiotti et al., in preparation (2010).

[15] J. Vines, E. E. Flanagan, arXiv:1009.4919 [gr-qc]].

[16] T. Damour and et al., Phys. Rev. D 77, 084017 (2008).

[17] T. Damour and A. Nagar, Physical Review D 76, 064028 (2007); Phys. Rev. D77, 024043 (2008).

[18] E. Schnetter, S. H. Hawley, and I. Hawke, Class. Quantum Grav. 21, 1465 (2004); D. Pollney, C. Reisswig, et al., Phys. Rev. D76, 124002 (2007); L. Baiotti, et al., Phys. Rev. D 71, 024035 (2005); B. Giacomazzo, and L. Rezzolla, Class. Quantum Grav. 24, S235 (2007).

[19] E. Gourgoulhon, P. Grandclément, K. Taniguchi, J. A. Marck, and S. Bonazzola, Phys. Rev. D 63, 064029 (2001). 